\title{
Convergent metatarsal fusion in jerboas and chickens is mediated by similarities and differences in the patterns of osteoblast and osteoclast activities
}

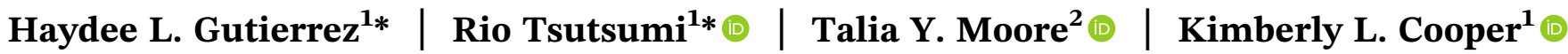

${ }^{1}$ Division of Biological Sciences, Section of Cellular and Developmental Biology, University of California San Diego, La Jolla, California

${ }^{2}$ Department of Ecology and Evolutionary Biology and Museum of Zoology, University of Michigan, Ann Arbor, Michigan

\section{Correspondence}

Kimberly L. Cooper, Division of Biological Sciences, Section of Cellular and Developmental Biology, University of California San Diego, La Jolla, CA 92093. Email: kcooper@ucsd.edu

\section{Funding information}

David and Lucile Packard Foundation, Grant/Award Number: 2015-63114; Pew Charitable Trusts, Grant/Award Number: 28635; Kinship Foundation, Grant/Award Number: 15-SSP-104

\begin{abstract}
In many vertebrate animals that run or leap, the metatarsals and/or metacarpals of the distal limb are fused into a single larger element, likely to resist fracture due to high ground-reaction forces during locomotion. Although metapodial fusion evolved independently in modern birds, ungulates, and jerboas, the developmental basis has only been explored in chickens, which diverged from the mammalian lineage approximately 300 million years ago. Here, we use a bipedal rodent, the lesser Egyptian jerboa (Jaculus jaculus), to understand the cellular processes of metatarsal fusion in a mammal, and we revisit the developing chicken to assess similarities and differences in the localization of osteoblast and osteoclast activities. In both species, adjacent metatarsals align along flat surfaces, osteoblasts cross the periosteal membrane to unite the three elements in a single circumference, and osteoclasts resorb bone at the interfaces leaving a single marrow cavity. However, the pattern of osteoclast activity differs in each species; osteoclasts are highly localized to resorb bone at the interfaces of neighboring jerboa metatarsals and are distributed throughout the endosteum of chicken metatarsals. Each species, therefore, provides an opportunity to understand mechanisms that pattern osteoblast and osteoclast activities to alter bone shape during development and evolution.
\end{abstract}

\section{K E Y W O R D S}

bipedalism, bone fusion, jerboa, limb evolution, osteoblasts, osteoclasts

\section{INTRODUCTION}

A diversity of limb skeletal forms support the weight of the body and allow a range of locomotor activities in vertebrate animals. In species that run or hop at high speeds, the metatarsals and/or metacarpals are often disproportionately elongated to increase stride length. However, simply lengthening a rod-like structure is accompanied by an

*Haydee L. Gutierrez and Rio Tsutsumi contributed equally to this work. increase in the likelihood of failure, or fracture, due to an increased bending moment with respect to a point load (e.g., an animal's foot striking the ground with metatarsals at an angle that is less than perpendicular to the ground). Vertebrate limbs have adopted a variety of bone densities, cortical thicknesses, and curvatures to compensate for increased length to maintain an optimal safety factor that balances stiffness while minimizing weight (Brassey, Kitchener, Withers, Manning, \& Sellers, 2013). In cases of extreme elongation in multiple lineages that include the birds, jerboas, and artiodactyls (e.g., camels and deer), metatarsals 
have fused to transform multiple thin rod-like elements into a single "cannon bone" with a larger outer diameter (Clifford, 2010; Mayr, 2016; Moore et al., 2015). Even if comprised of the same material properties with the same cortical thickness, the distribution of mass further from the center of the cross-section (neutral bending axis) would increase the second moment of area and the stiffness of the metatarsus (Koch, 1917). Therefore, a fused cannon bone would be expected to withstand higher bending forces than unfused metatarsals.

In vertebrates, the limb skeletal primordia emerge from within limb bud mesenchyme as small cartilage condensations. Each cartilage forms a template that is ultimately replaced by bone through the process of endochondral ossification. Chondrocytes in the center of the cartilage enlarge to become hypertrophic chondrocytes that are then replaced by osteoblasts to form the bony diaphysis, or shaft, with cartilage growth zones at each end of the skeletal element (Kronenberg, 2003). Together, osteoblasts and osteoclasts contribute to normal bone homeostasis, circumferential growth, and reshaping; osteoblasts have anabolic activity and deposit new bone whereas osteoclasts are catabolic cells that resorb mineralized bone matrix (Hadjidakis \& Androulakis, 2006).

While longitudinal growth of each limb bone is driven by the activity of growth cartilages, circumferential growth is driven by the activity of osteoblasts and osteoclasts that line the inner (endosteal) and outer (periosteal) surfaces of cortical bone. Radial growth is one type of circumferential growth of the long bone wherein periosteal mineral deposition is equally countered by resorption on the endosteal surface resulting in a relatively consistent cortical thickness. In contrast, preferential periosteal growth results in an increased cross-sectional thickness, while nonuniform circumferential growth alters the cross-sectional shape and/or produces a linear curvature (Allen \& Burr, 2014; Bateman, 1954).

Post-ossification fusion to unite the metatarsals into a single bone evolved independently in multiple vertebrate lineages, including bipedal hopping jerboas, the ancestor of modern birds, and artiodactyls that have additionally fused metacarpals. Both in mammals and in birds, the metatarsals first develop as individual cylindrical bones that later align and fuse the cortices into one bone that shares a single marrow cavity (Cooper et al., 2014; Lopez-Rios et al., 2014; Namba et al., 2010). Despite its frequent occurrence throughout vertebrate evolution, the developmental basis of bone fusion has been characterized only during fetal chicken development. However, the deep divergence of the synapsid (mammal) and sauropsid (dinosaurs including bird-like ancestors) lineages more than 300 million years ago means there is little reason to assume that the process of bone fusion is similar in birds and in mammals.
Here, we use the bipedal three-toed jerboa as a model to investigate the cellular mechanisms of bone fusion in a mammal. There are 33 species of jerboas including two genera of pygmy jerboas with metatarsals that do not fuse (Salpingotus and Cardiocranius), one species in which the three central metatarsals are partially fused (Euchoreutes naso), and a majority of jerboas with the three central metatarsals that fully fuse into a single cannon bone (Moore et al., 2015). The species that we study to understand the evolution of limb development, Jaculus jaculus, does not complete formation of the first and fifth digits during early embryonic development (Cooper et al., 2014). At birth, the remaining metatarsals of digits II, III, and IV are separate cylindrical bones. Soon after birth, while the metatarsals continue to rapidly elongate and grow radially, they fuse to form a single bone in the adult that trifurcates distally and articulates with each of the three proximal phalanges.

We describe the developmental process of metatarsal fusion in the jerboa in three steps: (a) alignment of the three metatarsals to form a transverse arch; (b) formation of mineralized bridges across the periosteum at the interface of adjacent bones; and (c) catabolism of all bone at the interfaces by localized osteoclast activity that unifies a single marrow cavity. To compare and contrast these cellular processes in jerboa with metatarsal fusion in birds (Namba et al., 2010), we also re-assessed chicken metatarsal fusion focusing on bone formation and resorption activities.

In both species, the interfaces of adjacent bone are closely abutted, and mineralized bridges form across the periosteal membrane to unite all three in a single cortical circumference. Bone is removed from the center to unify three distinct cavities into a single marrow space. There are, however, differences in the localization of anabolic (osteoblast) and catabolic (osteoclast) activities. During later phases of bone fusion in the jerboa, osteoblasts remain active at the interfaces of adjacent metatarsals, while osteoclasts are highly localized to degrade bone at the interfaces. In contrast, osteoclasts in the chicken are uniformly distributed around the circumference of all three skeletal elements. Our comparative anatomy and histology explain the structural reorganization of the distal limb to enable bipedal locomotion in the jerboas and in birds and establish the jerboa as a potential model to understand the uncoupling of anabolic and catabolic activities that shape the vertebrate skeleton during development and evolution.

\section{MATERIALS AND METHODS}

\section{1 | Animals}

Jerboas of the species $J$. jaculus were housed and maintained at the University of California San Diego as previously described (Jordan et al., 2011) and in full 
compliance with the Institutional Animal Care and Use Committee. Fertilized chicken eggs (Gallus gallus) were purchased from AA Lab Eggs, Inc. and incubated in a humidified rocking cabinet incubator at $37^{\circ} \mathrm{C}$ until P20 (hatching). Postnatal Day 2 and 5 (P2 and P5) chicken feet were purchased from Charles River Laboratories.

\subsection{Tissue processing}

Jerboas were anesthetized with $625 \mathrm{mg} / \mathrm{kg}$ ketamine $12.5 \mathrm{mg} / \mathrm{kg}$ xylazine by intraperitoneal injection and perfused with $20 \mathrm{ml}$ of phosphate-buffered saline (PBS) followed by $20 \mathrm{ml}$ of $4 \%$ paraformaldehyde (PFA) in PBS at room temperature. Metatarsals were then dissected and placed in 20\% sucrose $+4 \%$ PFA in PBS solution for $1.5 \mathrm{hr}$ rocking at $4^{\circ} \mathrm{C}$. Metatarsals were then embedded in SCEM media (cat C-EM001; Section-Lab) and frozen in a cryomold submerged in an isopentane and dry ice bath. Chicken embryos were collected and stages confirmed according to (Bellairs \& Osmond, 2005). Metatarsals were dissected and fixed overnight in 4\% PFA in PBS solution then placed in $30 \%$ sucrose in PBS solution rocking overnight at $4^{\circ} \mathrm{C}$. Metatarsals were then embedded in SCEM media (cat C-EM001; Section-Lab) and frozen in a cryomold suspended in an isopentane and dry ice bath.

Frozen specimen blocks were sectioned on a Leica Cryostat CM1950 using the CryoJane Tape-Transfer System. Adhesive Tape Windows (cat 39475214; Leica) were used to pick up the sections, which were then placed on slides coated with Solution B (cat 39475271; Leica). Slides were then exposed to two pulses, eight milliseconds each and at $360 \mathrm{~nm}$ of ultraviolet (UV) light, and the tape was removed.

\section{3 | Tartrate resistant acid phosphatase (TRAP) staining}

All chemicals used to stain for TRAP activity (Hadler et al., 2008) were purchased from Sigma. TRAP Basic Incubation Medium is comprised of $9.2 \mathrm{~g}$ sodium acetate anhydrous, $11.4 \mathrm{~g} \mathrm{~L}-(+)$ tartaric acid (cat 228729), $950 \mathrm{ml}$ distilled water, and $2.8 \mathrm{ml}$ glacial acetic acid (cat 695092) adjusted to $\mathrm{pH} 4.7-5.0$ with glacial acetic acid or $5 \mathrm{M}$ sodium hydroxide. Naphthol AS-BI phosphate substrate (cat AC415310010) is dissolved at $20 \mathrm{mg}$ per $\mathrm{ml}$ in ethylene glycol monoethyl ether (cat 128082). Sections were stained for TRAP activity by placing slides in prewarmed TRAP staining solution mix $(200 \mathrm{ml}$ TRAP basic incubation medium, $120 \mathrm{mg}$ Fast Red Violet LB Salt [cat F3381], and $1 \mathrm{ml}$ naphthol AS-BI phosphate substrate solution) at $37^{\circ} \mathrm{C}$ for $25 \mathrm{~min}$. Slides were then rinsed in distilled water, counterstained with $0.02 \%$ Fast Green (cat F7252) for 90 s, and rinsed in distilled water.
After dehydration through a graded series of EtOH, the slides were cleared in Xylenes and mounted in Permount media (cat SP15-100) under glass coverslips.

\subsection{Von Kossa staining}

One percent (1\%) aqueous silver nitrate solution was made by adding $1 \mathrm{~g}$ of silver nitrate (cat 56506-256; Sigma) to $100 \mathrm{ml}$ of distilled water. Five percent sodium thiosulfate solution was made by adding $5 \mathrm{~g}$ of sodium thiosulfate to $100 \mathrm{ml}$ of distilled water. $0.1 \%$ nuclear fast red solution was made by adding $0.1 \mathrm{~g}$ nuclear fast red (cat N0305; TCI) and $5 \mathrm{~g}$ aluminum sulfate (cat A1114; Spectrum) to $100 \mathrm{ml}$ distilled water and then boiled, cooled and filtered. A grain of thymol was added as a preservative. Sections were then warmed at $37^{\circ} \mathrm{C}$ for $15 \mathrm{~min}$, rinsed in PBS $2 \times 5 \mathrm{~min}$, and then rinsed in distilled water for $1 \mathrm{~min}$. Sections were then incubated in $1 \%$ silver nitrate solution in a glass staining dish under ultraviolet light (Spectronics Spectrolinker XL-1000 UV Crosslinker) for $25 \mathrm{~min}$. Slides were rinsed in distilled water for $1 \mathrm{~min}$, then placed in $5 \%$ sodium thiosulfate, then rinsed in distilled water for $1 \mathrm{~min}$. Slides were then counterstained in $0.1 \%$ nuclear fast red solution for $5 \mathrm{~min}$ and rinsed in distilled water. Slides were dehydrated $3 \mathrm{~min}$ in $95 \% \mathrm{EtOH}$ and $2 \times 3 \mathrm{~min}$ in $100 \% \mathrm{EtOH}$. Slides were then cleared in xylenes and coverslipped using Permount media (cat SP15-100; Fisher).

\subsection{Immunohistochemistry}

Slides were washed in PBS $2 \times 5 \mathrm{~min}$. Antigen retrieval was then performed by microwaving slides in $1 \times$ epitope unmasking buffer (cat 21760005-1; Bioworld) for 2 min at a temperature just below $100^{\circ} \mathrm{C}$ in a glass staining dish. Covered staining dish was then wrapped with a blue underpad and foil for $10 \mathrm{~min}$ at room temperature, placed at $4^{\circ} \mathrm{C}$ for $20 \mathrm{~min}$, then washed in PBS $2 \times 5 \mathrm{~min}$. Slides were placed in blocking solution consisting of $5 \%$ heatinactivated goat serum, $0.1 \%$ Triton (cat 100504-970; VWR), $0.02 \%$ SDS for $1 \mathrm{hr}$ at room temperature. Slides were then incubated with primary antibodies anti-Procollagen I (cat SP1.D8; DSHB) 1:20, anti-collagen XIVA1 (Novus NBP1-86877) 1:250, or anti-periostin 1:100 (cat ab14041; Abcam) diluted in block solution overnight at $4^{\circ} \mathrm{C}$. Slides were washed in PBS $+0.1 \%$ Triton $3 \times 10$ min. Slides were then incubated in the dark in Alexa Fluor secondary antibodies goat anti-mouse 488 (cat A21121; Life Tech) 1:500, goat anti-rabbit 594 (cat A11012; Life Tech) 1:500, and $1 \mu \mathrm{g} / \mathrm{ml} \mathrm{4',6-diamidino-2-phenylindole}$ (DAPI) (cat D1306; Life Tech) diluted in block solution for $1 \mathrm{hr}$ at room temperature. Slides were then washed in PBS $+0.1 \%$ Triton $3 \times 10 \mathrm{~min}$ and PBS $1 \times 5 \mathrm{~min}$ then 
coverslipped with Fluoromount G (cat OB10001; Southern Biotech) under glass coverslips. All images were taken on an Olympus BX61 compound microscope.

\subsection{Computed tomography}

Skeletal specimens for Cardiocranius paradoxus (MSB 199763) and E. naso (MSB 227347) were mounted in floral foam, and fluid specimens for J. jaculus, obtained from the colony at the Harvard Concord Field Station, were mounted in a plastic container in 95\% ethanol for micro-computed tomography (micro-CT) scanning. The specimens were scanned with a SkyScan 1173 micro-CT scanner (Bruker microCT, Kontich, Belgium). The specimens were scanned with $70 \mathrm{kV}$ voltage and $114 \mu \mathrm{A}$ current for all specimens except for the adult $J$. jaculus, which was scanned at $60 \mathrm{kV}$ and $113 \mu \mathrm{A}$. The specimens were scanned at resolutions resulting in $26.29 \mu \mathrm{m}$ (C. paradoxus), $22.3 \mu \mathrm{m}$ (E. naso), $16.34 \mu \mathrm{m}$ (adult J. jaculus), $35.53 \mu \mathrm{m}$ (juvenile J. jaculus) pixel sizes. The section images were reconstructed with the program NRecon and exported as 3D surface models. We segmented the surface models to isolate the metatarsals and digits and remove the proximal $50 \%$ of the metatarsals using MeshLab (Cignoni et al., 2008).

\section{3 | RESULTS}

\section{1 | The degree of metatarsal fusion among multiple species of bipedal jerboas correlates with increased adult body size}

The earliest diverging extant bipedal jerboas are the pygmy jerboas (subfamily Cardiocraniinae) with adult body weights that range from 6 to $13 \mathrm{~g}$ (Shenbrot, Sokolov, Heptner, \& Koval'skaya, 2008). These species have metatarsals that closely align in a transverse arch, but each metatarsal remains as a distinct skeletal element in adult animals (Figure 1a and Figure S1; Shenbrot et al., 2008; Smith et al., 2010). The next most recently derived morphotype is represented here by the long-eared jerboa (subfamily Euchoreutiinae, species E. naso) with metatarsals that are partially fused; the adult animals, which average about $30 \mathrm{~g}$ in body weight (Stubbe et al., 2007), have a contiguous bone marrow, but remnants of bone at the interfaces of adjacent metatarsals remain as columns that traverse the medullary cavity (Figure 1b). The fivetoed (subfamily Allactaginae) and three-toed jerboas (subfamily Dipodinae, including species J. jaculus and Dipus sagitta) are the most recently divergent; all have fully fused the three central metatarsals into a single cylindrical marrow cavity with no trace of the former interfaces between adjacent bones (Figure 1c,d), other than distally where the three trifurcate into the proximal phalanges.

Since metatarsals in quadrupedal rodents and in the earliest diverging pygmy jerboas are not fused, the transition from quadrupedalism to bipedalism most likely occurred in species with metatarsals that were also not fused. The unfused metatarsals of pygmy jerboas are disproportionately longer than those of closely related quadrupedal species (Moore et al., 2015), and they would have experienced a greater force per gram of animal while standing stationary by distributing weight among half as many feet. Furthermore, the shift from scurrying to hopping involves the addition of aerial phases, which produce much greater ground-reaction forces and bone stresses, even if the same number of legs are used in each gait (McMahon, Valiant, \& Frederick, 1987; Rubenson, Heliams, Lloyd, \& Fournier, 2004). We, therefore, hypothesize that the lack of fusion may have constrained the body size to maintain a safety factor that prevents metatarsal fracture. Indeed, increasingly fused metatarsals are accompanied by increases in body size; jerboas with fully fused metatarsals have reached a body size up to 415 grams in Allactaga major, two orders of magnitude greater than the pygmy species (Shenbrot et al., 2008). Since ground-reaction force and stress on elongated metatarsals increases with body size and with the addition of aerial phases in locomotion, this pattern suggests that metatarsal fusion may have been a structural adaptation that allowed body size to increase in these bipedal species.

\section{2 | Metatarsal fusion in $J$. jaculus proceeds through a series of events to reshape bone}

We describe the developmental process of complete metatarsal fusion in the crown group of jerboas that have fully fused metatarsals using $D$. sagitta for late gestational stages and $j$. jaculus for postnatal analyses (Moore et al., 2015; Pisano et al., 2015). In the midgestation jerboa embryo, metatarsal condensations of digits II and IV each angle away from the metatarsal of digit III (Figure 1e). By birth, the three metatarsals lie parallel to one another with the middle metatarsal in a slightly more dorsal position, and each has a circular shape in cross-section (Figure 1f,i). Within days after birth, the cross-sectional shape of each metatarsal has transformed such that the three collectively comprise a transverse arch (Figure 1j). The third metatarsal has adopted a "pie-wedge" shape in cross-section and serves as the "keystone," while the second and third metatarsals each have a more half-circular shape with a flat surface that lies adjacent to the central metatarsal. The neonatal 


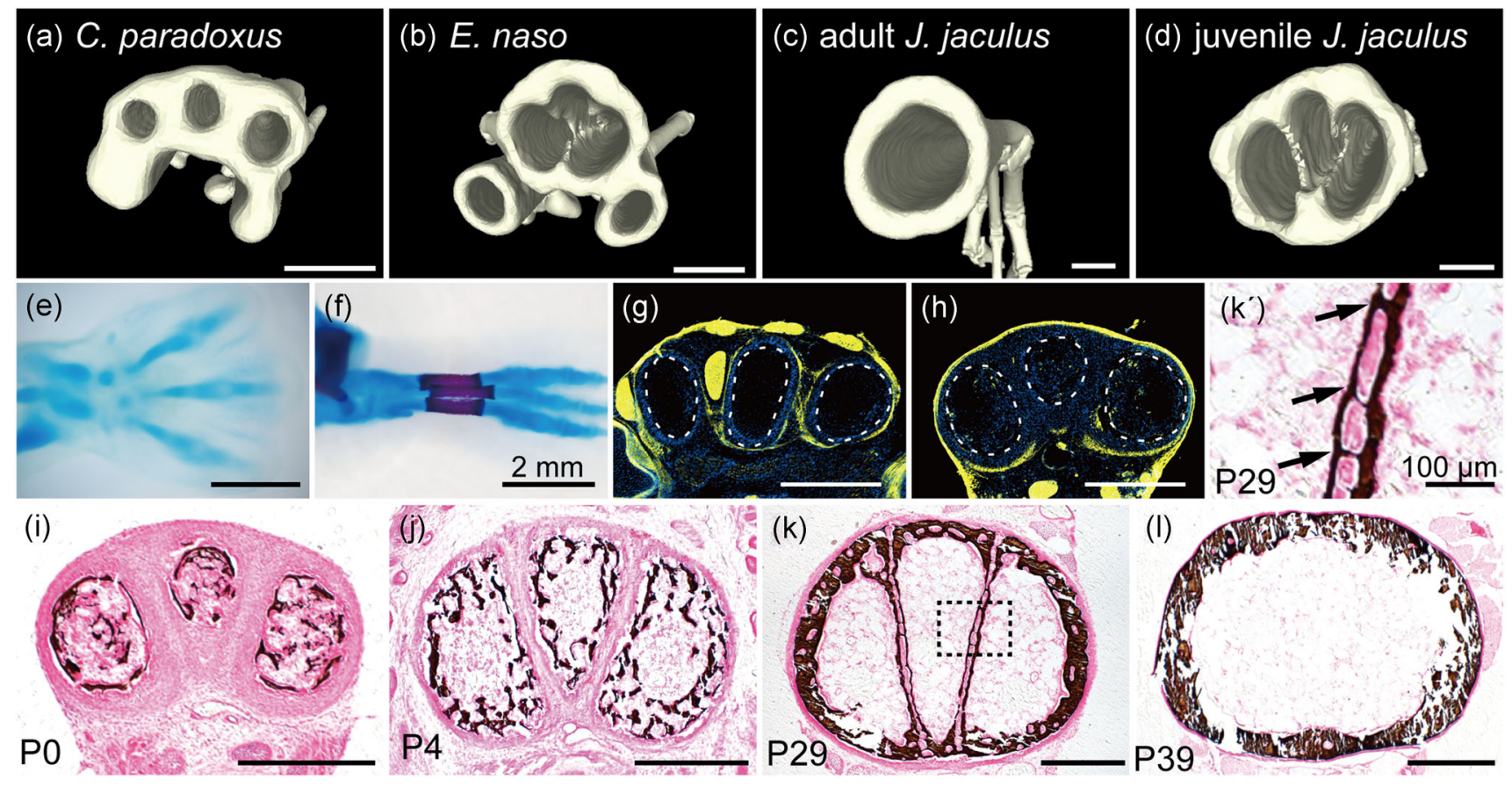

F I G U RE 1 Evolutionary and developmental process of alignment and fusion to unite three metatarsals into a single cannon bone in recently derived jerboas. microcomputed tomography showing cross-sections at approximately 50\% the length of metatarsus III of adult Cardiocranius paradoxus (a), Euchoreutes naso (b), adult jaculus jaculus (c), and juvenile J. jaculus (d). Note the CT scan resolution of C. paradoxus was insufficient for the rendered image to show the reported delineation between adjacent metatarsals that is more evident in Figure S1. Three-toed jerboa (Dipus sagitta) at approximately E13.5 (e) and P0 (f) stained with alcian blue and alizarin red. Immunostaining with anti-Collagen XIV (yellow) and DAPI (blue) on cross-sections of P0 jerboa (J. jaculus) hand (g) and foot (h). Dotted lines represent metapodial bones. While there are Collagen XIV positive layers between metacarpals in the hand, a Collagen XIV layer is absent between metatarsals in the foot ( $n=3$ each). (i-l) Temporal dynamics of cross-sectional shapes of metatarsals of the lesser Egyptian jerboa (J. jaculus) at P0 (i, $n=3)$, P4 (j, $n=3)$, P29 (k, $n=4)$, P39 $(1, n=3)$. The samples were stained by Von Kossa to detect mineralized bone in black and counterstained with nuclear fast red in pink. $\left(\mathrm{k}^{\prime}\right)$ The enlarged view of dotted-square in (k) at P29. Arrows show the bridging of three metatarsals. Each scalebar measures $500 \mu \mathrm{m}$ unless otherwise indicated in the figure. CT, computed tomography [Color figure can be viewed at wileyonlinelibrary.com]

state of three distinct metatarsals aligned into a transverse arch recapitulates the adult morphology of the earliest diverging pygmy jerboas (Figure 1a,i).

In J. jaculus, each of the three metatarsals is maintained as a discrete mineralized element for approximately three to 4 weeks after birth. The first evidence that fusion has begun is the appearance of mineralized bridges that invade the periosteal membranes and physically connect adjacent metatarsals (Figure 1k, $\mathrm{k}^{\prime}$ arrows). Mineralized bridging occurs not only dorsally and ventrally to unite the cortex that will ultimately encircle the unified marrow cavity, but also along the length of adjacent interfaces. Within 5-6 weeks after birth, all mineralized bone at these interfaces has been removed, and a single marrow cavity remains encircled by cortical bone (Figure 11).

Although the initiation of bridging and of bone catabolism does not occur at precise developmental ages or animal weights, their relative order is consistent and proceeds from proximal to distal over time. Transverse sections through an animal at P29 shows that catabolism of proximal bone is more similar to the midshaft of an older animal while the pattern of mineralized bridges more distally in the same individual is similar to the more proximal bone of a younger animal (Figure S2).

Since osteoblasts invade the periosteal membrane of adjacent metatarsals to form mineralized bridges, we suspected there may be a difference in the structure of the periosteum itself. In chicken, Collagen XIV, also known as Undulin, marks the outer layer of the periosteum (Bandyopadhyay, Kubilus, Crochiere, Linsenmayer, \& Tabin, 2008). In jerboa metacarpals that remain as distinct skeletal elements into adulthood, Collagen XIV expression encircles each skeletal element and is also strongly expressed in tendons and surrounding connective tissue (Figure 1g). In contrast, there is no expression of Collagen XIV between jerboa metatarsals in newborn animals (Figure 1h). Instead, a domain that may be the outer layer of the periosteum or a distinct layer of connective tissue, encircles all three skeletal elements. 


\section{3 | Spatial and temporal pattern of anabolic bone deposition by osteoblasts and catabolic bone resorption by osteoclasts in jerboa metatarsals}

With an understanding of the reorganization of mineralized bone over time, we next sought to determine if there is a correlated pattern of anabolic osteoblast activity. We performed immunofluorescence with an antibody to detect pro-Collagen I, the intracellular precursor of Collagen I, which is the major protein component of bone and is produced by osteoblasts (Prockop, Kivirikko, Tuderman, \& Guzman, 1979). In newborn jerboas, we saw pro-Collagen I expression in cells that completely encircle each of the three metatarsals in transverse sections (Figure 2a,b). However, by postnatal Day 21, the expression is discontinuous in the periosteum that lines the interface of adjacent metatarsals, though pro-Collagen I is present along the endosteal surface of the bone at the interfaces (Figure 2c, $\mathrm{c}^{\prime}, \mathrm{c}^{\prime \prime}$ arrow and arrowhead, respectively). We frequently observe no pro-Collagen I expression in the dorsal-most aspect of periosteum that lies between adjacent metatarsals (Figure $2 \mathrm{c}^{\prime}$, arrow).

The pattern of pro-Collagen I expression along interfaces between adjacent bones during the fourth week is somewhat surprising in light of the apparent
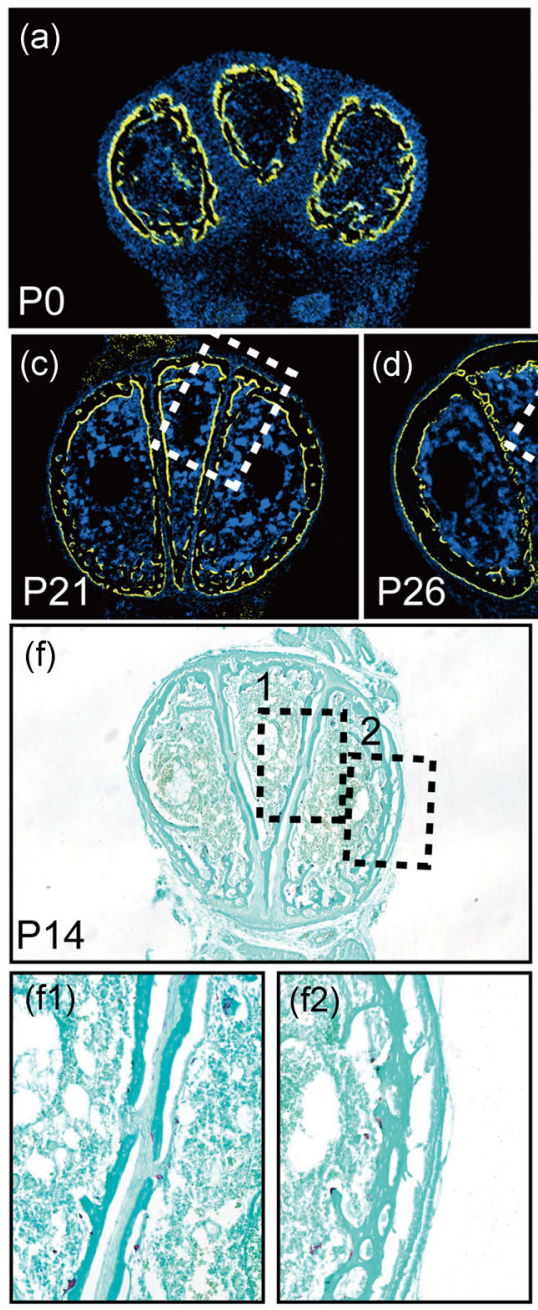
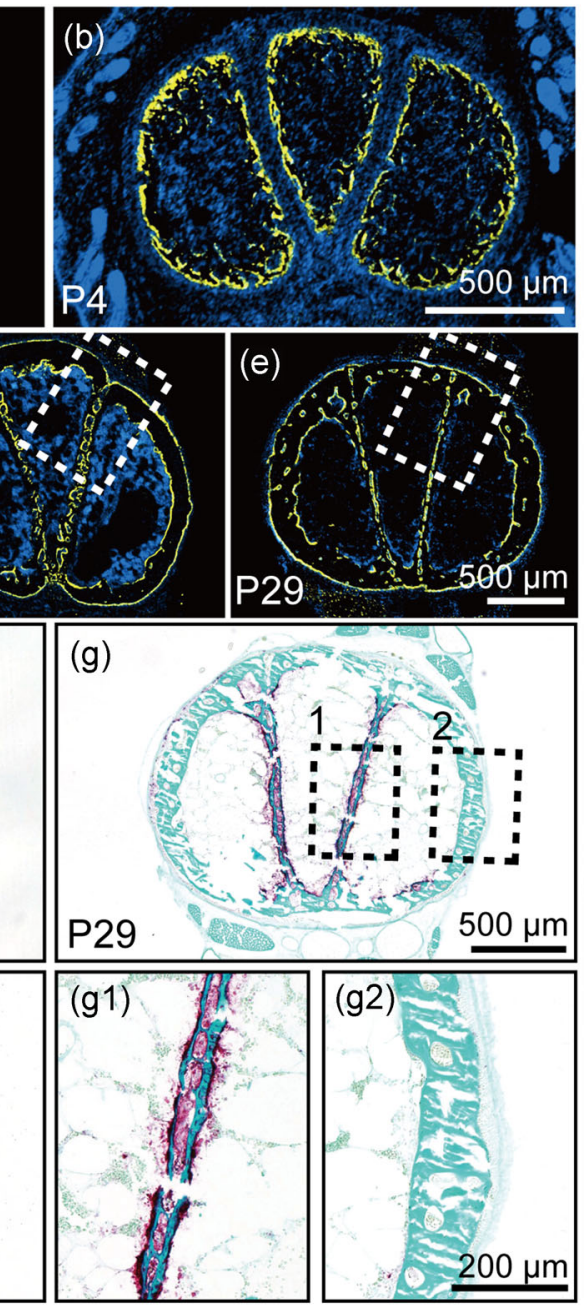
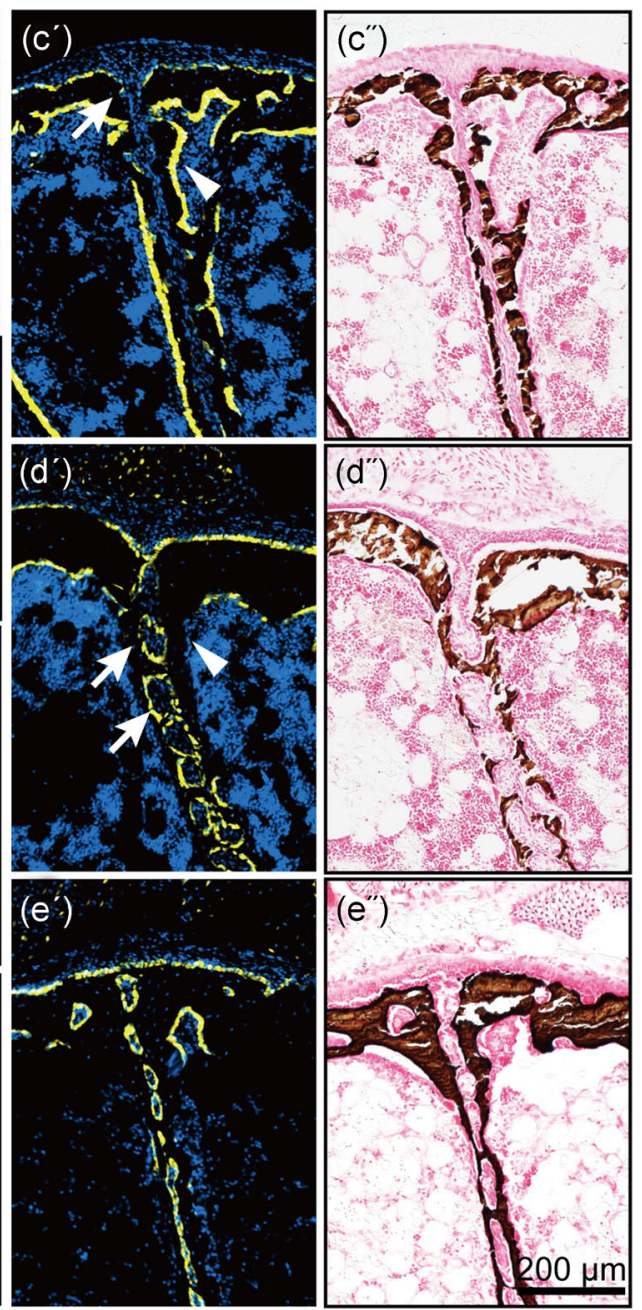

FIG URE 2 Temporal and spatial localization of osteoblasts and osteoclasts in metatarsals of jerboa (jaculus jaculus). Immunostaining using anti-pro-Collagen I (yellow) and DAPI (blue) of metatarsals in the lesser Egyptian jerboa at P0 (a, $n=5$ ), P4 (b, $n=4)$, P21 (c, $n=5$ ), P26 (d, $n=2)$, and P29 (e, $n=2)$. The enlarged views of the dotted rectangles in (c), (d), and (e) are shown in ( $\left.\mathrm{c}^{\prime}\right)$, ( $\left.\mathrm{d}^{\prime}\right)$, and $\left(\mathrm{e}^{\prime}\right)$. Von Kossa staining of corresponding serial sections are shown in $\left(\mathrm{c}^{\prime \prime}\right),\left(\mathrm{d}^{\prime \prime}\right)$, and $\left(\mathrm{e}^{\prime \prime}\right)$, respectively. Arrow in $\left(\mathrm{c}^{\prime}\right)$ denotes the absence of the proCollagen I in the dorsal-most periosteum, and the arrowhead shows expression in the endosteal layer. Arrows in $\left(d^{\prime}\right)$ show the expression of pro-Collagen I in the periosteum between metatarsals, and the arrowhead shows the absence of expression in the endosteal layer. TRAP staining of metatarsals at P14 (f, $n=4)$ and P29 (g, $n=4$ ) detects osteoclast activity in maroon counterstained with Fast Green. The enlarged views of the dotted rectangles are shown in (f1 and f2) and (g1 and g2), respectively. Scalebars denote measurements for groups of related panels. TRAP, tartrate-resistant acid phosphatase [Color figure can be viewed at wileyonlinelibrary.com] 
downregulation of pro-Collagen I expression in the periosteum by around 3 weeks after birth. At this later time point, expression becomes absent from the endosteum, instead of lines the periosteum, and in fact crosses the periosteum (Figure 2d, $\mathrm{d}^{\prime}$ arrowhead and arrows, respectively). This pattern of expression at 4 weeks is consistent with our observation that mineralized bridges cross the periosteum between adjacent metatarsals, indeed precedes the pattern of mineralization, and appears to encircle segments of the periosteum (Figure $2 \mathrm{~d}^{\prime \prime}$ ). To determine if cells within these loops of pro-Collagen I expressing cells retain periosteal identity, we also detected the expression of Periostin, an extracellular matrix protein expressed in connective tissues that include the periosteum. We find Periostin expression that is encircled by osteoblasts that line the mineralized bridges in the space between adjacent metatarsals as well as strong and contiguous Periostin expression that surrounds all three metatarsals (Figure S3a,b).

Osteoclast activity can be detected by enzyme chemodetection of TRAP. From soon after birth until near the end of the fourth postnatal week, there is very little osteoclast activity at the mid-diaphysis (Figure 2f,f1,f2). Beginning at around 4 weeks after birth, we observed high osteoclast activity associated with the bone that lies at the interfaces of the three metatarsals (Figure 2g,g1). In contrast, we observed very little TRAP activity associated with the outer cortical bone that encircles what is becoming a single marrow cavity (Figure 2g2). At this stage, there is still an expression of pro-Collagen I at the interface of the three metatarsals (Figure $\left.2 \mathrm{e}, \mathrm{e}^{\prime}, \mathrm{e}^{\prime \prime}\right)$. Together, this suggests that osteoclast differentiation or recruitment is localized to restrict bone resorption to the interfaces of adjacent bone within what will become a single marrow cavity.

\subsection{Osteoblast and osteoclast activities during fusion of the tarsometatarsus in chickens}

We showed that metatarsal fusion in jerboas occurs by locally altering the patterns of bone deposition and degradation through temporal and spatial differences in the anabolic activity of osteoblasts and the catabolic activity of osteoclasts. Bone fusion also occurs during late fetal development in all modern birds to unite the metatarsals of hindlimb digits II-IV into a single "tarsometatarsus" that also includes some of the tarsal, or ankle, elements. Considering the phenotypic convergence of metatarsal fusion in these two clades that diverged more than 300 million years ago, we next asked whether the independent evolution of metatarsal fusion occurred by similar spatial partitioning of osteoblast and/ or osteoclast activities.
Although the morphological process of tarsometatarsal fusion in chicken was previously well-described (Namba et al., 2010), we re-evaluated the shape transformation overtime to set the stage for our investigation of osteoblast and osteoclast activities. As in this prior work, Von Kossa staining of mineralized bone in transverse sections through the chicken foot at embryonic Day 13 (E13) revealed three distinct metatarsals each with a circular shape in crosssection through the midpoint of the diaphysis, the position where metatarsal fusion initiates in chicken (Figure 3a). By E15, the metatarsals of digits II and IV have adopted a halfcircular shape, and the metatarsal of digit III has become square at this position. Although the three metatarsals of the chicken foot lie in a plane alongside one another and do not form a transverse arch as in the jerboa, neighboring metatarsals do lie adjacent to one another along with flat interfaces in both chicken and jerboa (Figure 3b).

Chicken metatarsal cortical bone is more trabeculated, or spongy, and less compact than in the jerboa. As described by Namba and colleagues, the three metatarsals of the chicken become connected by trabeculae that form along the dorsal and ventral interfaces of metatarsals from Day 17 to Day 20 (Figure 3c,d). These trabeculae appear similar to the mineralized bridges that we observe crossing the periosteal membrane between adjacent jerboa metatarsals. Finally, from E20 to posthatching Day 5 (P5), mineralization at the interface of neighboring chicken metatarsals is removed leaving a single marrow cavity as in the jerboa (Figure 3d,e).

Also as in the jerboa, the expression pattern of proCollagen I appears to predict the structure of mineralized bone in the chicken tarsometatarsus. At E13, expression of pro-Collagen I in tissues surrounding the central metatarsal, which is more circular in its mineralized structure, is similar to the square shape of this skeletal element at E15 (Figure 3b,f). Although mineralized trabeculae do not bridge the space between adjacent metatarsals until about E17, proCollagen I expression starts to cross the periosteum by about E15 (Figure 3g,g', arrow). At E17, pro-Collagen I expression fully crosses the periosteum of adjacent metatarsals (Figure 3h, $\mathrm{h}^{\prime}$ arrows). At E20, pro-Collagen I expression surrounds the three metatarsals collectively, as the trabeculated bone is deposited around the circumference, and we sometimes observed downregulation of pro-Collagen I on the surface of the bone that remains at the interfaces, though this aspect of the phenotype is variable (Figure S4).

We next assessed whether osteoclast activity is localized to specifically resorb bone at the interfaces of adjacent metatarsals in chickens as in jerboas. In chickens, osteoclasts remain uniformly distributed around the endosteal circumference throughout the process of metatarsal fusion and are not enriched at the interfaces of adjacent metatarsals, in contrast with jerboas (Figure 3i,i1,i2). Even from E20 to P2 in the chicken, when the 
(a)

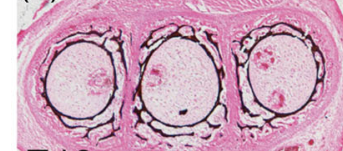

\section{E13}

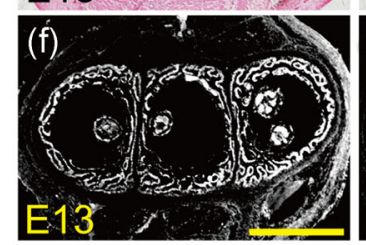

(b)

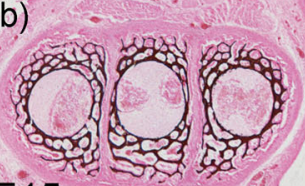

E15

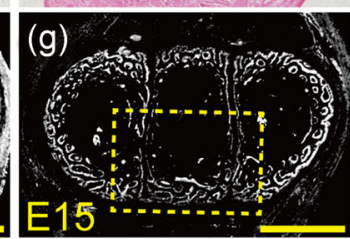

(c)

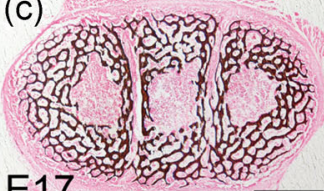

E17

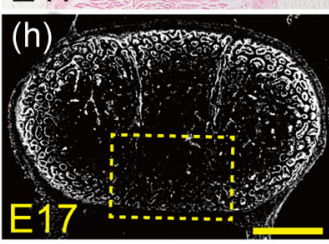

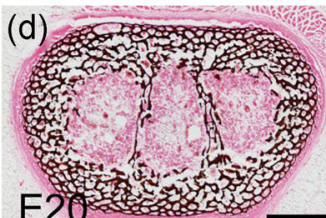

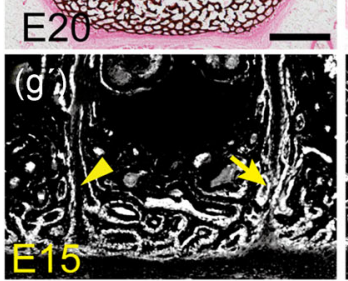

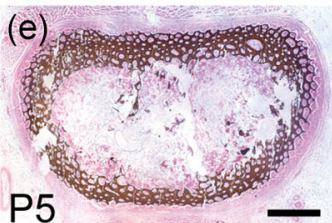

P5

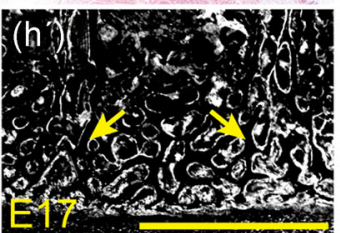

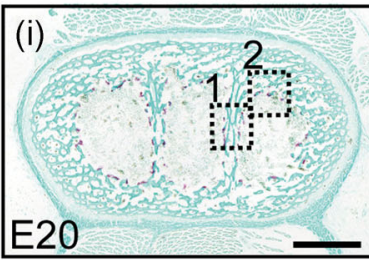

(i1)
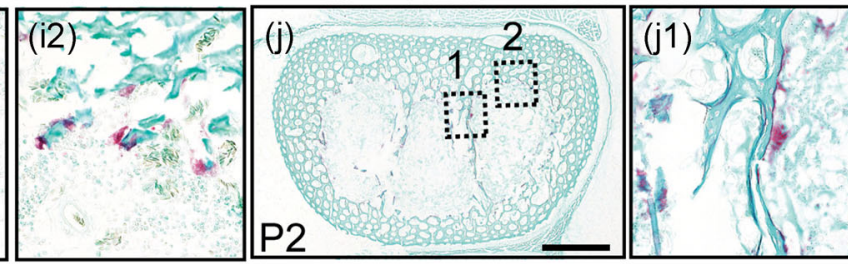

(j2)

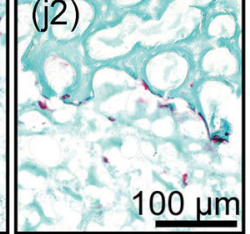

F I G U RE 3 Mineralized bone fusion and temporal and spatial localization of osteoblasts and osteoclasts in metatarsals of chicken (Gallus gallus). (a-e) Von Kossa staining of mineralized bone at E13 (a, $n=4)$, E15 (b, $n=4)$, E17 (c, $n=4)$, E20 (d, $n=4)$, and P5 (e, $n=1)$. (f-h') proCollagen I immunostaining (white) in serial sections at E13 (f, $n=4)$, E15 (g, $n=4)$, E17 (h, $n=4)$. The enlarged views of the dotted rectangles in (g) and $(\mathrm{h})$ are shown in $\left(\mathrm{g}^{\prime}\right)$ and $\left(\mathrm{h}^{\prime}\right)$, respectively. Arrowhead in $\left(\mathrm{g}^{\prime}\right)$ indicates a gap between Pro-Collagen I positive layers. Arrows in $\left(\mathrm{g}^{\prime}\right)$ and $\left(\mathrm{h}^{\prime}\right)$ show bridges of pro-Collagen I. (i-j') TRAP staining of tarsometatarsals in chicken at E20 (i, $n=4)$ and P2 (j, $n=2)$. The enlarged view of squares at the interface (1) and circumferential endosteum (2) in (i) and (j) are shown in adjacent panel insets. Scalebars measure $500 \mu \mathrm{m}$ for panels a-j, $\mathrm{g}^{\prime}$ and $\mathrm{h}^{\prime}$. Scalebars measure $100 \mu \mathrm{m}$ for insets i1, i2, j1, and j2. TRAP, tartrate-resistant acid phosphatase [Color figure can be viewed at wileyonlinelibrary.com]

bone is mostly resorbed between adjacent metatarsals, osteoclast activity never appears localized to these interfaces (Figure 3j,j1, j2). Therefore, the unification of a single marrow cavity in chickens occurs by uniform endosteal osteoclast activity rather than by the highly localized osteoclast activity that we observed in jerboas.

\section{DISCUSSION}

Here, we show that complete metatarsal fusion in the most derived jerboas, as well as in chickens, is characterized by localized alterations of anabolic osteoblast and catabolic osteoclast activities that change the pattern of mineralization in time and in space. Early in the process of bone fusion, the jerboa and chicken share a similar pattern of osteoblast activity that bridges the periosteum between adjacent metatarsals. The unusual behavior of osteoblasts that violate the periosteal boundary in both species is likely necessary to connect all three metatarsals in a single circumferential cortex. Once this is accomplished, osteoblast activity is sometimes decreased along with bone interfaces in the chicken but appears to persist in the jerboa periosteum until later stages when the bone at the interfaces is resorbed in both species.

In the final stage of metatarsal fusion in jerboas, a high density of osteoclast activity lines bone at the interface of adjacent metatarsals, and very few osteoclasts are associated with the endosteal surface that encircles what will be a single marrow cavity. It, therefore, appears that removal of bone from the center of the cannon bone is spatially localized. This contrasts with the chicken, in which osteoclasts appear uniformly and sparsely distributed along the endosteal surface of each skeletal element (Figure 4). If the bone is indeed resorbed uniformly from the endosteal surfaces of chicken metatarsals, it is possible that fusion is achieved, at least in part, by the difference in bone thickness in the two species. The cortical bone surrounding each of the three metatarsals is thin in the jerboa compared to the circumference of highly trabeculated bone that encircles the three elements of the chicken. The bone that lies between adjacent chicken metatarsals is also much thinner, perhaps in part due to decreased osteoblast activity before fusion. Uniform bone resorption of a cylinder with nonuniform thickness in the chicken would indeed preferentially remove thin bone at the interfaces, also because the interface is lined by osteoclasts flanking endosteal surfaces while the circumferential bone has only a single endosteal surface.

Before metatarsal fusion to form a single marrow cavity in either species, the three metatarsals achieve an intimate alignment that closely abuts adjacent surfaces along with flat interfaces. It is reasonable to assume that the close association of periosteal surfaces might be necessary to unify the three skeletal elements into a single cortex, but evidence from the jerboa lineage suggests that such a close association is not sufficient to trigger fusion. The very similar shape of a 


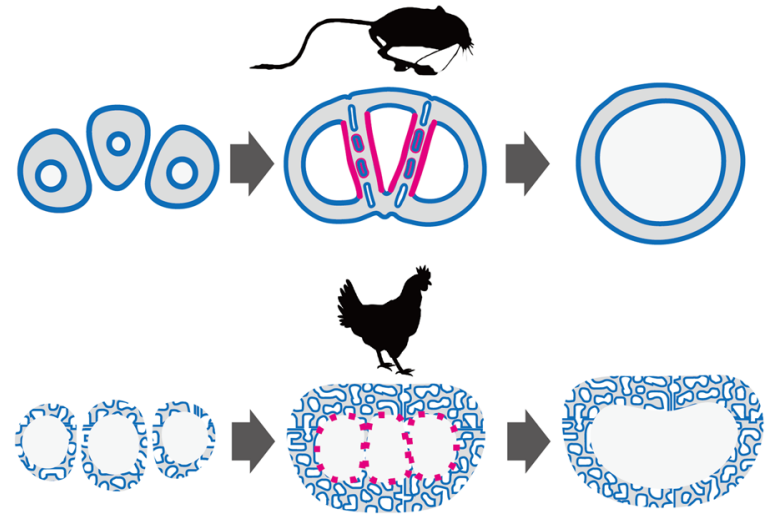

FIG URE 4 Schematic representation of jerboa and chicken metatarsals before, during, and after fusion. Blue depicts the location of osteoblasts that deposit bone. Magenta depicts the location of osteoclasts that resorb bone. While osteoclasts are highly localized to resorb bone at the interfaces of neighboring jerboa metatarsals, these catabolic cells are uniformly and sparsely distributed on the endosteum of chicken metatarsals throughout the process of bone fusion [Color figure can be viewed at wileyonlinelibrary.com]

transverse arch that we observe in neonatal jerboas of the species $J$. jaculus, is also present in adults of the pygmy jerboa species $C$. paradoxus (Moore et al., 2015). If close alignment along a flat surface were sufficient to allow passive fusion of adjacent cortical bones, the pygmy jerboa should not have three distinct metatarsals.

How is the locally biased pattern of osteoblast and osteoclast activity established to reshape mineralized bone for fusion? In other species, preferential periosteal growth is affected by mechanical stimuli applied to bones such that the shapes of bones are developmentally adapted to withstand load-bearing demands (Epker \& Frost, 1966; Frost, 2000; Levenston, Beaupré, \& Carter, 1998). In animals that develop without limb muscle or with muscle paralysis, absence of mechanical loading causes bones to retain a simpler columnar shape, and the mechanical resistance of bones is compromised (Sharir, Stern, Rot, Shahar, \& Zelzer, 2011). However, the process of bone fusion by mineralized bridge formation across the periosteal boundary and localization of osteoclast activity occurs before the time when juvenile jerboas adopt a quadrupedal gait at about 4 weeks after birth. Juvenile jerboas do not become fully bipedal until after metatarsal fusion is complete at about 5 weeks after birth (Eilam \& Shefer, 1997). Similarly, tarsometatarsal fusion in fetal chickens is almost complete before hatching (Namba et al., 2010). Therefore, bipedal locomotion does not seem to provide a mechanical stimulus that initiates metatarsal fusion, but rather fusion may be due to a developmental genetic program that is a result of natural selection to optimize the metatarsal safety factor.

It is interesting that osteoclast activity is locally upregulated at bone interfaces in the jerboa while osteoblast activity is sometimes locally downregulated at bone interfaces in the chicken. These data suggest that anabolic and catabolic activities can be uncoupled and spatially patterned in multiple ways to reshape skeletal elements, perhaps due to localized gene expression controlled by modular cis-regulatory elements. In mouse ribs, $G d f 5$ expression appears to be controlled by multiple enhancers that partition expression into subdomains along the circumference of the perichondrium (Guenther, Pantalena-Filho, \& Kingsley, 2008). Since the developmental process of metatarsal fusion in jerboas and chickens appears to be genetically determined, rather than induced by locomotory activities, these two species may be powerful systems to identify genes and associated cis-regulatory elements that partition the activities of osteoblasts and osteoclasts to define the adult bone shape. In addition to providing a basis for understanding the extraordinary diversity of limb bone shapes in vertebrate species, these studies would expand our understanding of two cell types that are critical regulators of bone growth and mineral homeostasis.

\section{ACKNOWLEDGMENTS}

We are grateful to Julio Rojano for assistance with cryosectioning. We thank all members of the Cooper laboratory as well as Drs. Michael Perry and James Posakony for thoughtful comments on the manuscript. This work was supported by a Searle Scholar Award from the Kinship Foundation, a Pew Biomedical Scholar Award from the Pew Charitable Trusts, and a Packard Fellowship in Science and Engineering from the David and Lucile Packard Foundation awarded to KLC. The authors declare that they have no affiliation or involvement in an organization or entity with a financial or non-financial interest in the subject matter or materials discussed in this manuscript.

\section{AUTHOR CONTRIBUTIONS}

H. L. G. performed the analysis of jerboa histology and immunostaining. H. L. G. and R. T. performed the analysis of chicken histology and immunostaining. T. Y. M. performed micro-CT of multiple species of jerboa metatarsals. R. T. and K. L. C. wrote the draft of the manuscript. All authors edited the manuscript. K. L.C. conceived of and oversaw the design, implementation, and analysis of the research.

\section{ORCID}

Rio Tsutsumi (D) http://orcid.org/0000-0001-9473-3923 Talia Y. Moore (1) http://orcid.org/0000-0003-0867-4512 Kimberly L. Cooper (D) http://orcid.org/0000-0001-58928838 


\section{REFERENCES}

Allen, M. R., \& Burr, D. B. (2014). Chapter 4-Bone modeling and remodeling. In D. B. Burr, \& M. R. Allen (Eds.), Basic and Applied Bone Biology (pp. 75-90). San Diego: Academic Press.

Bandyopadhyay, A., Kubilus, J. K., Crochiere, M. L., Linsenmayer, T. F., \& Tabin, C. J. (2008). Identification of unique molecular subdomains in the perichondrium and periosteum and their role in regulating gene expression in the underlying chondrocytes. Developmental Biology, 321, 162-174.

Bateman, N. (1954). Bone growth: A study of the grey-lethal and microphthalmic mutants of the mouse. Journal of Anatomy, 88, 212-262.

Bellairs, R., \& Osmond, M. (2005). Atlas of Chick Development. In 123Library, (Academic Press).

Brassey, C. A., Kitchener, A. C., Withers, P. J., Manning, P. L., \& Sellers, W. I. (2013). The role of cross-sectional geometry, curvature, and limb posture in maintaining equal safety factors: A computed tomography study. The Anatomical Record, 296, 395-413.

Cignoni, P., Callieri, M., Corsini, M., Dellepiane, M., Ganovelli, F., \& Ranzuglia, G. 2008. MeshLab: an Open-Source Mesh Processing Tool (The Eurographics Association).

Clifford, A. B. (2010). The evolution of the unguligrade manus in artiodactyls. Journal of Vertebrate Paleontology, 30, 1827-1839.

Cooper, K. L., Sears, K. E., Uygur, A., Maier, J., Baczkowski, K.-S., Brosnahan, M., ... Tabin, C. J. (2014). Patterning and postpatterning modes of evolutionary digit loss in mammals. Nature, 511, 41-45.

Eilam, D., \& Shefer, G. (1997). The developmental order of bipedal locomotion in the jerboa (Jaculus orientalis): Pivoting, creeping, quadrupedalism, and bipedalism. Developmental Psychobiology, 31, 137-142.

Epker, B. N., \& Frost, H. M. (1966). Biomechanical control of bone growth and development: A histologic and tetracycline study. Journal of Dental Research, 45, 364-371.

Frost, H. M. (2000). The Utah paradigm of skeletal physiology: An overview of its insights for bone, cartilage and collagenous tissue organs. Journal of Bone and Mineral Metabolism, 18, 305-316.

Guenther, C., Pantalena-Filho, L., \& Kingsley, D. M. (2008). Shaping skeletal growth by modular regulatory elements in the Bmp5 gene. PLoS Genetics, 4, e1000308.

Hadjidakis, D. J., \& Androulakis, I. I. (2006). Bone remodeling. Annals of the New York Academy of Sciences, 1092, 385-396.

Hadler, K. S., Huber, T., Cassady, A. I., Weber, J., Robinson, J., Burrows, A., ... Schenk, G. (2008). Identification of a non-purple tartrate-resistant acid phosphatase: an evolutionary link to Ser/ Thr protein phosphatases? BMC Research Notes, 1, 78.

Jordan, B., Vercammen, P., \& Cooper, K. L. (2011). Husbandry and breeding of the lesser Egyptian Jerboa, Jaculus jaculus. Cold Spring Harbor Protocols, 2011, 1457-1461.

Koch, J. C. (1917). The laws of bone architecture. American Journal of Anatomy, 21, 177-298.

Kronenberg, H. M. (2003). Developmental regulation of the growth plate. Nature, 423, 332-336.

Levenston, M. E., Beaupré, G. S., \& Carter, D. R. (1998). Loading mode interactions in simulations of long bone cross-sectional adaptation. Computer Methods in Biomechanics and Biomedical Engineering, 1, 303-319.

Lopez-Rios, J., Duchesne, A., Speziale, D., Andrey, G., Peterson, K. A., Germann, P., ... Zeller, R. (2014). Attenuated sensing of SHH by Ptch1 underlies evolution of bovine limbs. Nature, 511, 46-51.
Mayr, G. 2016. Avian Evolution: The Fossil Record of Birds and Its Paleobiological Significance (John Wiley \& Sons).

McMahon, T. A., Valiant, G., \& Frederick, E. C. (1987). Groucho running. Journal of Applied Physiology, 62, 2326-2337.

Moore, T. Y., Organ, C. L., Edwards, S. V., Biewener, A. A., Tabin, C. J., Jenkins, F. A., \& Cooper, K. L. (2015). Multiple phylogenetically distinct events shaped the evolution of limb skeletal morphologies associated with bipedalism in the jerboas. Current Biology, 25, 2785-2794.

Namba, Y., Yamazaki, Y., Yuguchi, M., Kameoka, S., Usami, S., Honda, K., \& Isokawa, K. (2010). Development of the tarsometatarsal skeleton by the lateral fusion of three cylindrical periosteal bones in the chick embryo (Gallus gallus). The Anatomical Record: Advances in Integrative Anatomy and Evolutionary Biology, 293, 1527-1535.

Pisano, J., Condamine, F. L., Lebedev, V., Bannikova, A., Quéré, J.-P., Shenbrot, G. I., ... Michaux, J. R. (2015). Out of Himalaya: The impact of past Asian environmental changes on the evolutionary and biogeographical history of Dipodoidea (Rodentia). Journal of Biogeography, 42, 856-870.

Prockop, D. J., Kivirikko, K. I., Tuderman, L., \& Guzman, N. A. (1979). The biosynthesis of collagen and its disorders. New England Journal of Medicine, 301, 13-23.

Rubenson, J., Heliams, D. B., Lloyd, D. G., \& Fournier, P. A. (2004). Gait selection in the ostrich: Mechanical and metabolic characteristics of walking and running with and without an aerial phase. Proceedings of the Royal Society of London. Series B: Biological Sciences, 271, 1091-1099.

Sharir, A., Stern, T., Rot, C., Shahar, R., \& Zelzer, E. (2011). Muscle force regulates bone shaping for optimal load-bearing capacity during embryogenesis. Development, 138, 3247-3259.

Shenbrot, G. I., Sokolov, V. E., Heptner, V. G., \& Koval'skaya, Yu. M. (2008). Jerboas: Mammals of Russia and Adjacent Regions. Enfield, NH: CRC Press.

Smith, A. T., Xie, Y., Hoffmann, R. S., Lunde, D., MacKinnon, J., Wilson, D. E., \& Wozencraft, W. C. (2010). A Guide to the Mammals of China (Princeton University Press).

Stubbe, A., Stubbe, M., Nyamsuren, B., Samjaa, R., Driechciarz, E., Driechciarz, R., ... Winter, M. (2007). Euchoreutes naso Sclater, 1890-ein SäugetierEndemit Zentralasiens. Erforschung Biologischer Ressourcen Der Mongolei, 10, 471-486.

\section{SUPPORTING INFORMATION}

Additional supporting information may be found online in the Supporting Information section.

How to cite this article: Gutierrez HL, Tsutsumi R, Moore TY, Cooper KL. Convergent metatarsal fusion in jerboas and chickens is mediated by similarities and differences in the patterns of osteoblast and osteoclast activities. Evolution \& Development. 2019;21:320-329. https://doi.org/10.1111/ede.12320 